\title{
Artificial Neural Network Approach for Solving Fuzzy Fractional order initial value problems under H-differentiability
}

\author{
Somayeh Ezadi ${ }^{1}$ and Tofigh Allahviranloo ${ }^{2}$ \\ ${ }^{1}$ Islamic Azad University Science and Research Branch Faculty of Basic Sciences \\ ${ }^{2}$ Bahcesehir Universitesi
}

December 13, 2020

\begin{abstract}
This paper aims to solve the celebrated Fuzzy Fractional Differential Equations (FFDE) using an Artificial Neural Network (ANN) technique. Compared to the integer order differential equation, the proposed FFDE can better describe several real application problems of various physical systems. To accomplish the aforementioned aim, the error back propagation algorithm and a multi-layer feed forward neural architecture are utilized using the unsupervised learning in order to minimize the error function as well as the modification of the parameters such as weights and biases. By combining the initial conditions with the ANN, output provides an appropriate approximate solution of the proposed FFDE. Then, two illustrative examples are solved to confirm the applicability of the concept as well as to demonstrate both the precision and effectiveness of the developed method. By comparing with some traditional methods, the obtained results reveals a close match that confirms both accuracy and correctness of the proposed method.
\end{abstract}

\section{Hosted file}

Artificial Neural Network Approach1.pdf available at https://authorea.com/users/382632/ articles/498539-artificial-neural-network-approach-for-solving-fuzzy-fractional-orderinitial-value-problems-under-h-differentiability 